Meta

Journal des traducteurs

Translators' Journal

\title{
Les formes sentencieuses : peut-on traduire la sagesse populaire?
}

\section{Jean-Claude Anscombre}

Volume 53, numéro 2, juin 2008

La traduction des séquences figées

The Translation of Frozen Sequences

URI : https://id.erudit.org/iderudit/018518ar

DOI : https://doi.org/10.7202/018518ar

Aller au sommaire du numéro

Éditeur(s)

Les Presses de l'Université de Montréal

ISSN

0026-0452 (imprimé)

1492-1421 (numérique)

Découvrir la revue

Citer cet article

Anscombre, J.-C. (2008). Les formes sentencieuses : peut-on traduire la sagesse populaire ? Meta, 53(2), 253-268. https://doi.org/10.7202/018518ar
Résumé de l'article

Le problème de la traduction d'une forme sentencieuse se heurte de façon cruciale à la définition de la classe des formes sentencieuses - dont les proverbes, les adages, les dictons, etc., ainsi qu'à celle d'éventuelles sous-classes. Après avoir proposé une classification des formes sentencieuses reposant sur des critères linguistiques, sont exposés et étudiés différents critères auxquels doit satisfaire une traduction de forme sentencieuse pour être adéquate. Différents exemples sont abordés, issus essentiellement du travail d'élaboration d'un dictionnaire franco-espagnol de formes sentencieuses par l'auteur de l'article.
Ce document est protégé par la loi sur le droit d'auteur. L'utilisation des services d'Érudit (y compris la reproduction) est assujettie à sa politique d'utilisation que vous pouvez consulter en ligne.

https://apropos.erudit.org/fr/usagers/politique-dutilisation/ 


\title{
Les formes sentencieuses: peut-on traduire la sagesse populaire?
}

\author{
JEAN-CLAUDE ANSCOMBRE \\ Université Paris XIII, Paris, France \\ anscombr@lli.univ-paris13.fr
}

\begin{abstract}
RÉSUMÉ
Le problème de la traduction d'une forme sentencieuse se heurte de façon cruciale à la définition de la classe des formes sentencieuses - dont les proverbes, les adages, les dictons, etc., ainsi qu'à celle d'éventuelles sous-classes. Après avoir proposé une classification des formes sentencieuses reposant sur des critères linguistiques, sont exposés et étudiés différents critères auxquels doit satisfaire une traduction de forme sentencieuse pour être adéquate. Différents exemples sont abordés, issus essentiellement du travail d'élaboration d'un dictionnaire franco-espagnol de formes sentencieuses par l'auteur de l'article.
\end{abstract}

\section{ABSTRACT}

When intending to translate sententious sayings, one crucially meets the problem of the lack of operative definitions as far as the class - and possible subclasses - of sententious forms are concerned. After providing a classification of sententious sayings based on linguistic criteria, this study investigates which conditions are to be fulfilled for an adequate translation of these forms. Several examples are analyzed, mainly borrowed from the author's experience in elaborating a bilingual French-Spanish dictionary of sententious sayings.

\section{MOTS-CLÉS/KEYWORDS}

forme sentencieuse, adages, proverbes, espagnol, problèmes de traduction

[...] Manier savamment une langue, c'est toujours pratiquer une espèce de sorcellerie évocatoire...

(Baudelaire, L'Art romantique).

\section{Introduction}

Je voudrais dans cette brève étude exposer certains problèmes auxquels on se trouve confronté dès lors qu'on se frotte à la traduction de ce type particulier de construction que sont les formes sentencieuses, et plus particulièrement les proverbes - je préciserai plus loin le sens exact qu'ont pour moi ces deux termes. Bien que n'étant pas traducteur professionnel, il m'est arrivé en plusieurs circonstances relevant de l'écrit, mais tout aussi bien de l'oral, d'avoir à affronter ce problème. Préoccupation qui m'est à l'heure actuelle quotidienne dans l'élaboration d'un dictionnaire bilingue espagnol-français des formes sentencieuses. C'est donc en quelque sorte du fruit de ma petite expérience (et de mes nombreux déboires!) dont je désire faire part dans cette petite étude.

\section{Formes sentencieuses, proverbes, etc.: quelques définitions}

La plupart des auteurs se consacrant à l'étude des formes sentencieuses (proverbes, dictons, adages, sentences, etc.), ne se posent guère de questions «théoriques", et le 
domaine se caractérise par une terminologie aussi floue quabondante. Parmi les termes le plus fréquemment rencontrés, je ne mentionnerai ici que les principaux, à savoir: adage, apophtegme, aphorisme, dicton, maxime, précepte, sentence, proverbe, etc. Les recueils contemporains, en total désaccord entre eux, nous proposent la plupart du temps des classements fantaisistes sous des appellations encore plus fantaisistes. Considérons en effet la très célèbre forme sentencieuse Une hirondelle ne fait pas le printemps. P. DesRuisseaux la classe dans les proverbes (comme le Grand Robert et le TLF), alors que A. Pierron la considère comme un dicton. Quant à Maloux, il la classe dans les proverbes du grec ancien - bien que cette forme sentencieuse existe dans à peu près toutes les langues indo-européennes contemporaines ${ }^{1}$. Delacourt y voit une maxime, Djavadi parle de dicton météorologique, et, prudent, Rey-Chantreau ne la classe pas, non plus que Lis et Barbier, qui y voient une «observation ». F. Montreynaud, A. Pierron et F. Suzzoni renvoient pour l'origine à Le Roux de Lincy ${ }^{2}$, qui renvoie à son tour à un auteur du Xvi s., et ce, malgré l'existence du modèle latin pourtant bien connu Una hirundo non effecit ver. P. DesRuisseaux voit un proverbe dans Nous n'avons pas gardé les cochons ensemble, y compris dans la variante Est-ce que nous avons gardé les cochons ensemble?, et également dans La vie n'est pas rose, et Dournon range Revenons à nos moutons dans les expressions devenues proverbiales! Et si certains auteurs présentent des traductions de proverbes étrangers - selon des choix discutables et des traductions souvent encore plus discutables, c'est la plupart du temps en partant de l'hypothèse implicite que la traduction d'un proverbe d'une langue doit être un proverbe dans l'autre langue, et ce, à n'importe quel prix. Hypothèse qui n'est qu'un cas particulier de l'hypothèse plus générale qu'un proverbe, quelles que soient sa région et son époque d'origine, sera à jamais proverbe: Once a proverb, always a proverb. D'où des équivalences douteuses, ainsi celle-ci, trouvée dans un recueil récent: En bouche close jamais mouche n'entra, proverbe $\mathrm{du} \mathrm{XVII}^{\mathrm{e}}$ s. totalement désuet aujourd'hui, et pourtant présenté comme l'équivalent contemporain de l'espagnol En boca cerrada no entran moscas, celui-là bien vivant de nos jours.

La critique est facile, dit-on. Ce désordre est l'indice d'un problème de fond, et je vais donc tenter d'y mettre un peu d'ordre.

Une première remarque sera que les phénomènes qui nous occupent ici ont trait à des constructions phrastiques. De plus, ces constructions phrastiques ont une propriété qui les distingue d'autres constructions phrastiques: à l'intérieur d'un discours donné, elles sont généralement mobiles, et font partie de ce que les linguistes appellent des phrases autonomes. On peut par exemple, dans un discours comme Les dés sont jetés, inutile d'insister, rejeter la phrase sentencieuse en position finale sans changement notable de sens: Inutile d'insister, les dés sont jetés. Une seconde remarque sera que toutes les phrases autonomes ne méritent pas le statut de phrase sentencieuse: ainsi une phrase comme Les voitures ont quatre roues est également autonome ${ }^{3}$, et ne semble guère sentencieuse. À l'intérieur donc des phrases autonomes, je distinguerai les phrases sentencieuses par le critère de combinabilité avec la tournure Comme dit $X, X$ étant l'auteur présumé de la phrase autonome. Par exemple:

(1) Comme (le) dit La Rochefoucauld, on ne blâme le vice et on ne loue la vertu que par intérêt.

(2) Comme on dit, il faut battre le fer quand il est chaud. 
Ce critère distingue bien deux sous-classes parmi les phrases autonomes, puisqu'on n'aura jamais:

(3) ${ }^{\star}$ Comme on dit, les voitures ont quatre roues.

Bien qu'anodine, cette remarque n'est pas sans intérêt, puisqu'elle permet éventuellement de repérer dans un texte source donné qu'une phrase est sentencieuse, et qu'on doit par conséquent lui rechercher une équivalence sentencieuse dans la langue cible. Il est assez fréquent, en effet, que des commentaires «méta-linguistiques ${ }^{4}$ de ce type figurent dans les textes, ainsi:

(4) Pero - decía un refrán español - «Hasta que pasa el rabo todo es toro...» (Arturo Pérez-Reverte, La reina del Sur, Éd. Alfaguara, 2002, p. 154)5.

(5) Mais comme le dit le vieil adage "Méfiez-vous de l'eau qui dort»! En effet, au printemps, lorsque la neige fond, elle libère des polluants...» (Le journal du CNRS, $\mathrm{n}^{\circ} 172$, mai 2004, p. 11).

Tout traducteur, même ignorant le sens exact de Méfiez-vous de l'eau qui dort, saura d'entrée de jeu, en vertu de la présence de comme dit le vieil adage, qu'il s'agira d'en trouver une traduction sentencieuse.

Parmi les phrases sentencieuses ainsi définies - autonomes et combinables avec comme dit $X$, j'en distinguerai deux types. Le premier correspond à un auteur précis - ainsi La Rochefoucauld dans l'exemple ci-dessus: c'est le domaine des maximes, sentences, morales, qui puise habituellement dans l'histoire et la littérature attachées à la langue considérée. Je ne m'en occuperai pas ici, et réserverai mes efforts au second cas, celui de l'auteur anonyme, que les linguistes ${ }^{6}$ ont baptisé du curieux nom de ON-locuteur. Ce ON-locuteur est fréquemment désigné par la sagesse des nations, la sagesse populaire, le bon sens populaire, etc. En voici quelques exemples empruntés à diverses langues:

(6) La mariée était trop belle.

(7) Mieux vaut un mauvais accord qu'un bon procès.

(8) A friend in need is a friend indeed.

(9) It takes two to tango.

(10) No hay mal que por bien no venga.

(11) No hay moros en la costa.

(12) Eine Schwalbe macht noch keinen Sommer.

(13) Wie der Vater, so der Sohn.

(14) Tal farás, tal trobarás.

(15) Una flor no fa estiu.

Notons qu'une forme sentencieuse peut selon le cas être vue comme ayant un auteur spécifique ou étant le fait d'un ON-locuteur. Ainsi, S = Rien ne sert de courir, il faut partir à point, peut être citée comme provenant de La Fontaine, ou comme issue de la sagesse populaire. De tels cas sont relativement fréquents, dans la mesure où il y a de constants et nombreux échanges entre la parole "populaire» et la parole «littéraire». Notons qu'il y a cependant des différences entre les deux emplois. Supposons par exemple que mon ami Max fasse preuve d'une activité fébrile: je pourrais l'inciter à plus de calme en disant: "Tu as tort de te précipiter: comme l'a dit La Fontaine, 
rien ne sert de courir, il faut partir à point. » On notera que dans cet exemple le présent comme le dit La Fontaine est moins naturel, dû au fait que $S$ tend à être perçu comme le fait d'un $\mathrm{ON}$-locuteur, La Fontaine n'en étant plus que l'ancien auteur.

À l'intérieur des formes sentencieuses à $O N$-locuteur, je distinguerai encore deux sous-classes. Celle des formes sentencieuses qui sont génériques - elles représentent une vérité valable extra tempore, et celle des formes sentencieuses qui ne le sont pas. À ces dernières, on réserve le nom de phrases situationnelles. Elles qualifient directement une situation, et en font partie des choses comme La mariée est trop belle, Un ange passe, Il y a de l'eau dans le gaz,... Elles sont en fait événementielles, et portent ou peuvent porter des marques circonstancielles, ce qui n'est pas le cas des sentencieuses génériques. On comparera de ce point de vue:

(16) Attention, dans cette maison les murs ont des oreilles.

(17) Tout le monde s'est tu. Un ange est passé.

Et:

(18) ${ }^{\star}$ Attention, dans cette maison, une hirondelle ne fait pas le printemps.

(19) *Tout le monde s'est tu. Qui n'a dit mot a consenti.

À l'ensemble des phrases sentencieuses ayant un $\mathrm{ON}$-locuteur et de nature générique, je réserverai le nom de phrases parémiques. Cette dernière catégorie sera à son tour divisée en plusieurs sous-classes, selon certaines caractéristiques:

a) La sous-classe des tautologies: ce sont des phrases de la forme Un sou est un sou, angl. Duty is duty, all. Mann ist Mann, cat. El diner ès diner, esp. Un día es un día, ital. Domenica è sempre domenica, etc. On les reconnaît essentiellement à une structure superficielle très souvent proche de ' $p$ est $p$ '.

b) La sous-classe des phrases parémiques exhibant certains schémas rythmiques, ellemême divisée en deux sous-classes, selon que la phrase est métaphorique ou non. La première classe, celle des [proverbe], correspond grosso modo aux «proverbes » au sens habituel, la seconde est celle des [adage] et des [dicton] ${ }^{7}$.

c) Le reste, i.e. la sous-classe des phrases parémiques n'ayant pas d'autre(s) propriété(s) saillante(s).

Sans entrer dans des détails techniques qui n'ont pas d'intérêt ici, voici quelques exemples. Considérons par exemple le bien connu Un mauvais arrangement vaut mieux qu'un bon procès. Elle se combine sans problème avec Comme on dit, et elle est assez visiblement générique - on peut la combiner avec souvent/toujours sans en trahir l'esprit. Il s'agit donc d'une phrase parémique, dont la structure superficielle n'est pas celle d'une tautologie. Le découpage Un mauvais arrangement/Vaut mieux qu'un bon procès (suggéré par le parallélisme mauvais arrangement/bon procès dans GN et GV) montre une structure rythmique 6/6. Cette phrase parémique n'est pas métaphorique, il s'agit donc d'un [adage]. La pluie du matin n'arrête pas le pèlerin découpée selon GN/GV comme précédemment montre une structure 5/7 et une rime -in/-in qui en fait cette fois un [dicton]. Considérons maintenant Qui va à la chasse/ perd sa place: il exhibe une rime -as/-as sur une structure $5 / 3^{9}$, et est métaphorique: c'est bien un [proverbe]. Enfin, Chacun voit midi à sa porte n'a pas de caractéristique particulière en dehors de la métaphoricité: c'est une simple phrase parémique. Notons pour terminer cette longue introduction aux structures parémiques que les cloisons entre les différentes catégories ne sont pas étanches, et qu'il arrive qu'on passe de l'une à l'autre. 
Résumons cette classification établie de façon sommaire par un schéma:

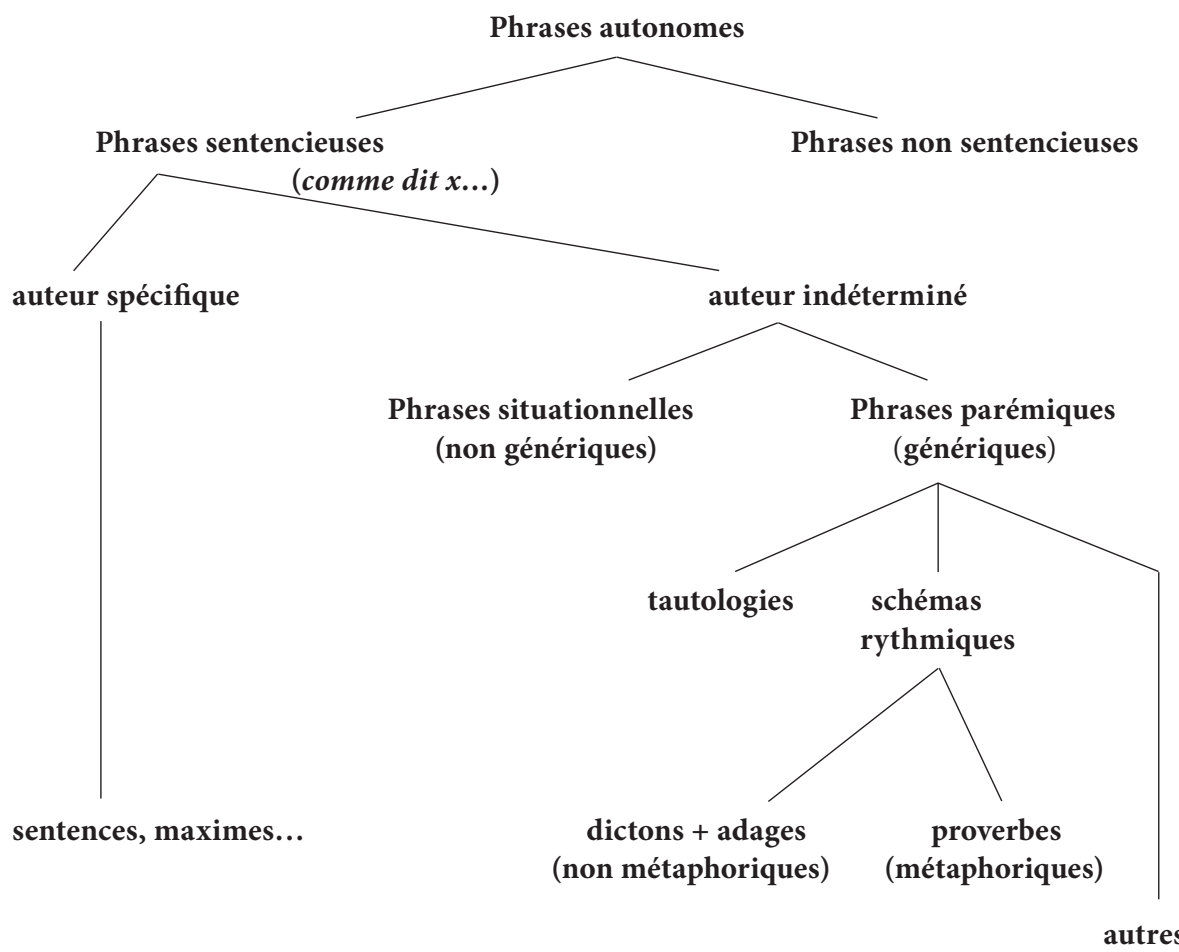

Le problème qui m'occupe ici peut alors se formuler de la façon suivante: étant donné une forme sentencieuse $\mathbf{S}$ d'une langue source, comment lui faire correspondre au mieux une forme sentencieuse $\mathbf{S}$ " d'une langue cible? Comme annoncé, j'utiliserai principalement l'espagnol et le français comme sources et cibles.

\section{Dessine-moi une équivalence entre formes sentencieuses}

\subsection{Le fonds commun}

Le premier réflexe de tout traducteur non professionnel - ce qui est mon cas - lorsqu'il lui faut fournir une équivalence d'une forme sentencieuse est bien entendu de chercher "quelque chose qui ressemble». Cette démarche est quelque peu naïve: il est assez exceptionnel que traduire se réduise à calquer, y compris et surtout dans le domaine sentencieux, et pour certaines raisons qui apparaîtront plus loin. Elle n'est cependant pas totalement dépourvue de sens, du moins à l'intérieur du champ indoeuropéen. En effet, l'utilisation massive du latin comme langue véhiculaire ainsi que l'importance de la culture classique gréco-latine dans notre enseignement ont fait que le domaine sentencieux indo-européen provient majoritairement d'un fonds commun latin, que ce soit au niveau de l'idée de base, ou même de la forme. Voici un cas assez étonnant, où les langues indo-européennes semblent avoir pratiquement copié le modèle latin. Il s'agit de Una arunda non effecit ver "Une hirondelle ne fait pas le printemps", qui outre l'équivalent français a donné: esp. Una golondrina no 
hace verano; cat. Una orenata no fa estiu; ital. Una rondine non fa primavera; angl. One swallow does not make a summer; all. Eine Schwalbe macht noch keinen Sommer; suéd. En svala gør ingen sommar; port. Uma andorinha não faz primavera; gall. Unha andorinha soa non fai verán; russe Odna lastochka vesnij ne delaet; grec moderne

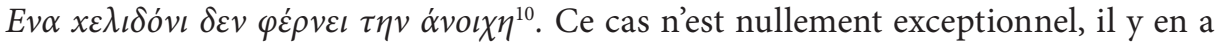
d'autres: ainsi, sur le modèle latin de Canes qui plurimum latrant, perraro mordent, on a formé en français Chien qui aboie ne mord pas, Perro ladrador poco mordedor en espagnol, Gos que lladra no mossega en catalan, $A$ barking dog never bites en anglais, Can che abbaia non morde en italien, Cão que ladra não morde en portugais, etc. En cas donc de "panne de traduction», on peut effectivement penser à aller chercher l'original latin correspondant à la forme sentencieuse de la langue source, lequel peut suggérer une équivalence dans la langue cible. Voici un exemple où effectivement ce procédé donne un résultat positif. Considérons la très connue forme sentencieuse espagnole Zapatero, a tus zapatos, lit. "Cordonnier, à tes chaussures", et supposons pour la démonstration que nous ayons un certain mal à trouver une correspondance en français langue cible. L'original latin - si l'on en croit du moins Pline l'Ancien, est Ne sutor ultra crepidam "Cordonnier, (ne va) pas au-delà de la sandale», version latine de la réplique supposée du peintre Apeles à un cordonnier qui critiquait un de ses tableaux. Le sens devient alors plus clair: le cordonnier n'y joue qu'un rôle accessoire, l'ensemble étant une version polie de «Occupe-toi de tes affaires», ou encore de "Femme, à tes casseroles». Il s'agit d'une forme sentencieuse destinée à faire savoir que la compétence de chacun ne peut ni ne doit dépasser sa sphère d'activité. Or, la sphère d'activité comporte essentiellement le métier, ce qui suggère immédiatement la correspondance À chacun son métier, et les vaches seront bien gardées.

Ce procédé, s'il n'est pas à rejeter, possède cependant ses limites. La ressemblance de surface peut n'être précisément que de surface - les apparences sont parfois trompeuses - même si les deux formes sentencieuses comparées proviennent d'un unique modèle latin. Reprenons ainsi l'exemple ci-dessus Chien qui aboie ne mord pas, l'origine latine en étant Canes qui plurimum latrant, perraro mordent. On aurait donc tendance à voir dans l'espagnol Perro ladrador, poco mordedor ${ }^{11}$ la parfaite équivalence cherchée. Et on aurait tort: la forme sentencieuse espagnole possède en effet deux sens, d'une part "une menace bruyante est rarement une véritable menace», sens attesté entre autres dans les zones nord et est de l'Espagne. Mais aussi «ceux qui parlent beaucoup agissent rarement», sens commun dans la zone de la capitale ${ }^{12}$, et qui correspond en fait au français Les conseilleurs ne sont pas les payeurs, et surtout à l'anglais All talk and no action.

On voit où est le problème: certes, c'est bien d'une certaine façon une ressemblance que cherche le traducteur. Mais nous n’avons pas défini ce qu'était une ressemblance ou, du moins, nous avons limité son champ d'application à la seule surface des phrases sentencieuses à l'étude.

Nous allons maintenant nous efforcer de délimiter la notion de ressemblance/ équivalence, aussi indispensable qu'elle est floue. Pour ce faire, je vais tenter de définir différents types d'équivalence, en en illustrant l'application et aussi les limitations. 


\subsection{L'équivalence catégorielle}

Une première idée de l'équivalence, et qui vient assez naturellement à l'esprit, est l'idée de l'équivalence catégorielle. Elle signifie qu'à une forme sentencieuse d'une certaine catégorie, on doit s'efforcer de faire correspondre une forme sentencieuse de la même catégorie. Outre l'idée de ressemblance, c'est aussi l'idée de fonctionnalité semblable qui prévaut ici. En effet, le fonctionnement des différentes catégories sentencieuses n'est pas le même. Ainsi, les phrases situationnelles comme Il a passé de l'eau sous les ponts ou C'est bonnet blanc et blanc bonnet se contentent de la simple appréciation d'une situation, alors que les tautologies ont souvent un côté prescriptif, ainsi Un sou est un sou ou Trop, c'est trop. Prescriptifs aussi les adages comme Un mauvais arrangement vaut mieux qu'un bon procès ou Jeux de mains, jeux de vilains, ainsi que les dictons agricoles À la Saint-Rémi, cueille tes fruits, ou À la SainteCatherine, tout prend racine. En revanche, les dictons météorologiques se présentent comme des observations: Petite pluie abat grand vent, ou Noël au balcon, Pâques aux tisons. Les proverbes enfin présentent ce qu'il est convenu d'appeler des doxa, i.e. des schémas de raisonnement ${ }^{13}$. Concluons: pour ne pas se tromper de fonctionnement lors de la recherche d'équivalences, mieux vaut les chercher à l'intérieur de la même catégorie. Certes, il ne manque pas, à l'intérieur du domaine indo-européen qui m'occupe ici, de cas où l'équivalence peut effectivement avoir lieu à l'intérieur d'une même catégorie. Prenons par exemple la phrase sentencieuse Il y a anguille sous roche. Il s'agit d'une phrase situationnelle, reconnaissable au fait qu'elle peut être combinée avec un déictique comme $L \grave{a}$, et qu'il y a possibilité de variations temporelles:

(20) «...Un privé...Il n’y avait pas nécessairement anguille sous roche. Il pouvait fort bien, en dehors de son boulot, connaître une admiratrice de Gil Andréa...» (Léo Malet, M'as-tu-vu en cadavre?, Fleuve noir, 1998, p. 195).

Or l'espagnol possède une phrase sentencieuse de même sens, à savoir Hay gato encerrado ${ }^{14}$, qui a le bon goût d'être également situationnelle, comme le montre l'attestation suivante:

(21) «...éste utilizaba el formal término milord para dirigirse al herido. Allí había gato encerrado...» (Arturo y Carlota Pérez-Reverte, El Capitán Alatriste I, Ed. Alfaguara, 1998, p. 88).

où l'expression en question, outre qu'elle est accompagnée du déictique allí = 'là', est de plus fléchie à l'imparfait. Malheureusement, tout n'est pas pour le mieux dans le meilleur des mondes, et ce cas idyllique ne saurait être considéré que comme une heureuse exception. Trop souvent, le traducteur de phrases sentencieuses devra se résoudre à changer de catégorie s'il veut fournir une équivalence plausible. Considérons ainsi la phrase sentencieuse, cette fois espagnole et fort courante, Agua pasada no mueve molino ${ }^{15}$, qui signifie grosso modo que les choses passées ont perdu toute pertinence. Il s'agit d'une expression métaphorique, et son schéma rythmique /'-/-'-/-'-/-'-/, presque complètement régulier, montre qu'il s'agit donc d'un proverbe. Le problème réside en ce qu'il n'y a rien d'équivalent en français, et le traducteur devra donc choisir entre deux possibilités: traduire notre phrase par Le passé est le passé, qui conserve l'idée, mais perd la métaphore de l'espagnol, et de plus change de catégorie: la phrase française est en effet une tautologie. Or, cette tautologie existe aussi en espagnol, à savoir El pasado, pasado está. Ou alors se résigner à 
rendre l'original par Laissons les morts enterrer les morts, qui est métaphorique et présente une pseudo-rime (morts/morts), mais n'a pas la généralité de la tournure espagnole, qui peut s'appliquer à des circonstances plus frivoles que sa consœur française. Autre problème: comme bien souvent dans le domaine sentencieux, l'équivalence, y compris catégorielle, peut recéler des pièges redoutables. Soit par exemple le cas de Un sou est un sou, visiblement une tautologie qu'il s'agit de rendre en espagnol. À première vue, rien de plus facile: l'espagnol possède justement une phrase sentencieuse, La pela es la pela ${ }^{16}$, qui semble convenir parfaitement à tous points de vue. C'est une tautologie, apparemment de même sens que l'original, et qui pousse même l'amabilité jusqu'à être familière, tout comme l'original. En fait, la forme sentencieuse espagnole se prononce habituellement avec un $l$ rétroflexe à l'imitation de la prononciation catalane, et pour se moquer des catalans et de leur amour supposé de l'argent. Une traduction plus exacte de La pela es la pela serait donc en fait Un chou c'est un chou, ce qui nous laisse sans véritable équivalent pour Un sou est un sou. On peut cependant s'en tirer avec Il n'y a pas de petit profit, qui présente le défaut de ne pas être une tautologie, mais une simple phrase parémique sans caractéristiques particulières.

On voit ainsi apparaître le fond du problème: certes, l'équivalence catégorielle est importante, mais elle est, à y regarder de plus près, d'une plus grande complexité que prévu. C'est que bien des facteurs entrent en jeu, et l'équivalence catégorielle n'est que l'un d'entre eux, qui a trait au fonctionnement en discours. En d'autres termes, une catégorie correspond à un mode d'insertion et d'articulation dans le discours, et donc au paramètre pragmatique. Mais d'autres équivalences sont envisageables, et doivent même être envisagées.

\subsection{D’autres équivalences}

Un problème qu'on rencontre assez vite concerne ce que j'appellerai l'équivalence lexicologique. Elle a trait au problème du figement dans le monde parémique. D’une façon générale, les phrases sentencieuses ne sont pas figées, c'est-à-dire que le figement n'est pas un trait qui les caractérise ${ }^{17}$. On peut le voir à de nombreuses propriétés, la plus apparente étant l'existence, y compris pour un même locuteur, de variantes. Ainsi, la très connue forme $\bar{A}$ cheval donné on ne regarde pas la bride admet entre autres variantes $A$ cheval donné, ne lui regarde pas en la bouche (Dournon); $A$ cheval donné, on ne regarde pas à la bouche (DesRuisseaux, Maloux, TLF); À cheval donné, on ne regarde pas à la bride (TLF, Gd Robert); À cheval donné, on ne regarde pas la bouche (Delacourt); $A$ cheval donné, on ne regarde pas les dents (Lis et Barbier). Ce qui n'empêche pas qu'on rencontre dans la catégorie des phrases sentencieuses comme dans toutes les catégories - des éléments qui sont figés. Il semble par exemple n'y avoir aucune variante répertoriée à À cœur vaillant, rien d'impossible. Un traducteur peut donc parfaitement estimer qu'une traduction soignée doit fournir pour une phrase sentencieuse figée une phrase également figée. Cette louable intention ne dépasse malheureusement jamais le niveau du vœu pieux, pour des raisons qui ne dépendent pas de la volonté du traducteur. L'une d'entre elles provient des influences constantes et réciproques entre la langue parlée et les écrits littéraires. Dans le cas du français, un exemple typique est celui des Fables de La Fontaine. La Fontaine n'a pas inventé les morales de ses fables, et n'a fait que reprendre des expressions populaires. 
Ainsi la célèbre La raison du plus fort est toujours la meilleure se trouve dès le $\mathrm{XVI}^{\mathrm{e}}$ siècle sous la forme Où force règne, raison n'a lieu, adage très vraisemblablement adapté du latin médiéval Tunc jus calcatur, violentia cum dominatur ${ }^{18}$. Le succès rencontré par les Fables a fait que la langue parlée a recueilli la morale de La Fontaine sans la modifier, ce qui est assez fréquent. L'équivalent espagnol Donde fuerza viene, derecho se pierde, bien qu'ayant également un auteur prestigieux ${ }^{19}$, ne bénéficie plus de cette aura, et a développé plusieurs variantes. Par ailleurs, le choix de rendre une forme figée par une forme figée peut se heurter à d'autres exigences d'équivalence, en particulier l'équivalence statistique et l'équivalence stylistique. Chaque langue ayant son propre génie, ce qui est courant dans l'une peut être rare, voire inexistant dans l'autre, et vice-versa. Considérons par exemple Il n'y a pas de fumée sans feu. Certes, l'espagnol possède la phrase parémique No hay humo sin fuego: mais alors que l'original français est d'un emploi banal et courant, son analogue espagnol l'est beaucoup moins, et «sonne» un peu livresque. On utiliserait beaucoup plus spontanément pour traduire l'idée recherchée Cuando el río suena, agua lleva ${ }^{20}$. Choisir cette dernière expression serait donc faire prévaloir l'équivalence statistique. Autre exemple: l'espagnol En la variedad está el gusto, d'un usage très banal. Il n'y a en fait rien d'équivalent en français, si ce n'est L'ennui naquit un jour de l'uniformité, d'une utilisation nettement plus limitée que son analogue français. Si l'on veut respecter l'équivalence statistique, il faudra se rabattre sur des tournures au caractère parémique peut-être moins marqué, mais d'une fréquence d'apparition comparable à celle de la leçon espagnole. Par exemple Il faut savoir varier les plaisirs, dont le statut de phrase parémique n'est pas assuré: certains sujets parlants hésitent devant la combinaison Comme on dit, il faut savoir varier les plaisirs.

Le cas de L'ennui naquit un jour de l'uniformité nous met sur la piste d'une autre équivalence, à savoir l'équivalence stylistique. Cette phrase sentencieuse montre en effet, outre un vocabulaire assez «culte», un usage du passé simple tout à fait exceptionnel, et qui a été presque totalement remplacé par le présent ${ }^{21}$, hormis quelques rares survivants ${ }^{22}$. L'équivalence stylistique consistera donc à fournir une équivalence qui respecte le niveau stylistique, lequel se manifeste selon deux axes: un premier concerne le niveau de langue. Il peut être culte, neutre, familier, argotique, grossier, etc. Le problème est que chaque langue définit ces niveaux à l'intérieur de son système propre, et qu'il n'y a pas - ou pas nécessairement - correspondance entre niveaux stylistiques d'une langue à l'autre. Alors que l'espagnol admet sans problème d'utiliser des mots grossiers, le français est plus réticent sur ce point, et se contentera sans doute du niveau familier. L'argot est plus facilement employé dans le langage de tous les jours en français qu'en espagnol, où la séparation entre les deux niveaux est plus nette. D'où une recherche de correspondance de niveaux qui, dans le cas du style, est assez souvent délicate. En voici un exemple, la phrase sentencieuse Quien quiera peces, que se moje el culo, lit. "Qui veut des poissons, qu'il se mouille le c....». Or si le mot culo est d'un usage banal en espagnol - il entre dans de nombreuses tournures familières - il est nettement plus marqué en français, où il est senti comme grossier et surtout vulgaire. Il s'agira donc de trouver un équivalent dans le style familier qui évite le piège de vouloir à tout prix utiliser l'équivalent français de culo. Ce qui écarte d'entrée de jeu Qui veut la fin veut les moyens, qui d'une part, est d'un niveau stylistique très au-dessus du niveau familier requis, et d'autre part, fait table rase de la métaphore présente dans l'original. Le second axe de l'équivalence stylistique concerne les 
variantes régionales. Certaines variantes sont en effet identifiées comme caractéristiques d'une région, ou simplement marquées comme 'régionales'. Variation qu'on peut utiliser pour rendre certaines «atmosphères ». Supposons par exemple un roman paysan espagnol où l'un des protagonistes déclare que Entre col y col lechuga ${ }^{23}$ "Il faut savoir varier les plaisirs». Bien entendu, si l'esprit de la traduction est correct, la forme en revanche détonne dans l'atmosphère paysanne supposée. Et ne parlons pas de «L'ennui naquit un jour de l'uniformité»... Heureusement, la nature (linguistique) fait bien les choses: il existe en français un Changement d'herbage réjouit les veaux qui respecte l'équivalence sémantique in situ de façon me semble-t-il plus satisfaisante. On voit ainsi apparaître une nouvelle équivalence, l'équivalence sémantique. Il ne s'agit évidemment pas que les deux expressions source et cible aient le même sens, cette exigence allant de soi. Il s'agit de l'idée que ce sens soit atteint si possible par le même procédé dans les deux langues: à une métaphore on tentera donc de faire correspondre la même métaphore, ou du moins une métaphore relevant du même champ. Dans le domaine indo-européen, marqué par une culture de base commune, transmise par le latin, et contrôlée par une religion commune, la tâche n'est pas surhumaine. Le fonds métaphorique est fréquemment partagé, ce qui ne se produit plus dans des cultures ayant d'autres origines. En particulier pour ce qui est des métaphores animales: elles sont typiques d'une culture, et un proverbe comme Corneille dans la main vaut mieux qu'aigle en l'air ${ }^{24}$, malgré une évidente parenté avec Más vale pájaro en mano que ciento volando et $A$ bird in the hand is better than two in the bush, ne passera jamais pour un proverbe indo-européen. Ni la corneille, ni l'aigle ne sont des familiers de notre parc animalier parémique. Cette équivalence sémantique fera préférer Más vale un toma que dos te daré25 à Más vale pájaro en mano que ciento volando comme traduction de Un tiens vaut mieux que deux tu l'auras, sur la base des métaphores présentes, bien que la seconde parémie espagnole soit plus courante que la première, et qu'on ne satisfait donc pas le critère d'équivalence statistique. Pour des raisons identiques, on pourra penser à traduire Il ne faut pas vendre la peau de l'ours avant de l'avoir tué par No hay que vender la piel del oso antes de haberlo cazado ${ }^{26}$, plutôt que par No hay que comerse el conejo antes de haberlo cazado $^{27}$. Cette seconde parémie étant de toutes façons moins courante que la première, on satisfait de plus - au moins partiellement - l'équivalence statistique.

Je vais maintenant aborder le délicat problème de l'équivalence rythmique. L'observation et l'analyse montrent qu'en fait, le trait parémique le plus saillant est la présence d'une structure rythmique. En d'autres termes, toute langue possède, prêts à l'emploi, un certain nombre de patrons rythmiques qui servent à construire des phrases sentencieuses. Ils sont en petit nombre, et se retrouvent également dans les comptines, les slogans, et partiellement dans les structures onomatopéiques. Ils correspondent à des moules poétiques caractéristiques d'une langue donnée, et la poésie habituelle n'a fait qu'utiliser ces moules en les épurant ${ }^{28}$. Ainsi, la phrase sentencieuse Bonne renommée vaut mieux que ceinture dorée peut se décomposer en Bonne renommée/vaut mieux que/ceinture dorée, c'est-à-dire un tercet de type a(4) b(3) a (4) ${ }^{29}$, avec de plus une structure rythmique de type /'- -'/'- '/'- -'/, parfaitement symétrique. L'espagnol Aunque la mona se vista de seda, mona se queda ${ }^{30}$ est également un tercet $\mathrm{a}(5) \mathrm{b}(6) \mathrm{b}(5)$, de structure rythmique /'- -'-/ -'- -'-/' - -'-/, schéma également symétrique. Bien entendu, il s'agit là de cas d'école, il y a des cas moins réguliers. L'importance de ces structures rythmiques se manifeste de différentes façons. D'une 
part, les sujets parlants classent plus volontiers dans les proverbes les phrases sentencieuses ayant une structure rythmique que celles n'en ayant pas. Ils en arrivent même à identifier comme "proverbes» des phrases sentencieuses inventées de toutes pièces, mais pourvues d'une structure rythmique de bon aloi. D'autre part, du point de vue diachronique, les formes sentencieuses empruntées à un auteur sont souvent remodelées pour s'ajuster à un patron métrique. Ainsi, La critique est facile, mais l'art est difficile, distique a(6) a(6), a été refait avec rime à partir d'un original La critique est aisée, et l'art est difficile, sans rime. L'espagnol Perro ladrador, poco mordedor, distique également, de structure a(6) a(6), est passé par différentes étapes antérieures, entre autres Perro ladrador, nunca muerde $=\mathrm{a}(6) \mathrm{b}(4)$, et Perro ladrador, nunca buen mordedor $=\mathrm{a}(6) \mathrm{a}(7)$.

On imagine aisément quel va être le problème. Si le traducteur désire conserver ce petit air authentique que confère la présence d'une structure rythmique, il lui faudra chercher une traduction possédant également une structure rythmique, et conforme aux schémas de la langue cible, qui n'ont aucune raison d'être identiques à ceux de la langue source, et encore moins d'apparaître simultanément ou non dans des formes sentencieuses équivalentes. On aura une idée de la difficulté dans le cas du passage de l'espagnol au français et vice-versa, si l'on considère que l'apparition de l'alexandrin dans la poésie française a eu une influence considérable sur le plan des patrons rythmiques du monde parémique, phénomène qui ne s'est pas produit pour l'espagnol, qui montre donc une plus grande variété dans les rythmes.

\section{Sans un peu de travail, on n'a point de plaisir: quelques cas épineux}

Comme on se l'imaginera sans peine, le travail du traducteur de phrases sentencieuses n'est pas facile, il doit tenter de concilier les exigences des différentes équivalences que nous venons de passer en revue. Certes, il y a des cas idylliques: ainsi, à l'espagnol De tal palo, tal astilla, métaphorique et de structure rythmique a(4) b(4), correspond un Tel père, tel fils, également métaphorique, distique également de forme a(2) a(2), les phrases espagnole et française étant toutes deux fort courantes, et du même niveau stylistique neutre habituel. Mais la plupart du temps, le traducteur devra dans le cas qui nous occupe ici se contenter d'une cote mal taillée, voire pire: on ne peut pas contenter tout le monde et son père!

Je vais donc terminer cet exposé par l'examen de quelques cas réels spécialement ardus, qui montreront qu'en toute dernière extrémité, c'est la sensibilité linguistique du traducteur plutôt que ses aptitudes techniques à la traduction qui fourniront une possible équivalence. De tels cas ne sont nullement l'exception, et comme nous allons le voir, la difficulté réside non dans l'existence ou non d'une correspondance quand on passe d'une langue à l'autre, mais dans le fait que très souvent cette correspondance n'est pas une «bonne» correspondance, et foule au pied l'ensemble des équivalences que j'ai cru pouvoir dégager plus haut.

Commençons par le très connu Cuando las barbas de tu vecino veas pelar, echa las tuyas a remojar, lit. 'Quand tu verras qu'on rase la barbe de ton voisin, met la tienne à tremper', forme sentencieuse conseillant de prendre ses précautions quand le danger s'approche. Son statut sentencieux ne fait aucun doute, non plus que son caractère métaphorique. Par ailleurs, son patron rythmique est tout à fait remarquable: 
/Cuando las barbas/de tu vecino/veas pelar/echa las tuyas/a remojar/, c'est-à-dire a(5) $\mathrm{b}(5) \mathrm{a}(5) \mathrm{c}(5) \mathrm{a}(5)$. C'est un quintil, et la disposition abaca est grosso modo celle du genre espagnol romancillo. Or, il n'y a rien d'équivalent en français contemporain. La parémie parfois proposée Si on rosse ton voisin, prépare tes reins, métaphorique et de structure a(6) a(4), est complètement sortie de l'usage contemporain et fait plutôt sourire. On n'a donc d'autre solution que de sortir du domaine restreint des proverbes, et de chercher un équivalent dans les phrases sentencieuses tout court. Bien entendu, cette solution du désespoir ne respecte à peu près aucune équivalence, si ce n'est la simple équivalence fonctionnelle. En fait, la valeur d'avertissement est assez bien rendue par la phrase situationnelle Attention! Ça n'arrive pas qu'aux autres, de création récente. Notons que le processus inverse se produit tout aussi bien: à une phrase source sans caractéristique saillante correspond une phrase cible d'une souscatégorie bien particulière. Considérons ainsi la phrase sentencieuse La beauté ne se mange pas en salade et tentons de lui trouver un équivalent en espagnol. Elle n'a pas de trait particulier, hormis la métaphore «alimentaire» attachée à manger en salade: la seule beauté d'une femme ne fait pas bouillir la marmite. Il nous faut donc chercher une métaphore alimentaire attachée à la beauté féminine. Le hasard faisant bien les choses, l'espagnol possède la phrase parémique Con hermosura sola, no se pone la olla ${ }^{31}$, qui correspond parfaitement pour le sens, présente également une métaphore alimentaire et est de même niveau stylistique, familier en l'occurrence. Mais elle possède de plus une structure rythmique de distique isosyllabique a(7) a(7), absente de l'original: c'est un proverbe. Soit maintenant la très courante phrase sentencieuse No todo el monte es orégano, lit. 'Tout le mont n'est pas de l'origan', signifiant qu'il ne faut pas s'attendre à ce que tout soit facile. La facilité est ici liée à l'origan, plante médicinale célèbre pour ses vertus aromatiques. Là se trouve la difficulté: si l'origan est relativement connu en France, il ne fait pas partie des plantes de ce type dont le nom vient spontanément aux lèvres. La solution consiste donc à chercher une plante odorifère aussi populaire en France que l'origan l'est en Espagne, et pouvant de plus être associée à la facilité. On peut penser à la rose, chantée par tous, et qui nous fournit une possible équivalence avec Tout n'est pas rose dans la vie.

Un sous-domaine sentencieux qui se révèle assez catastrophique pour la recherche d'équivalence, est celui des dictons agricoles et météorologiques. Les raisons en sont claires: les dictons agricoles et météorologiques d'une part s'appuient sur le calendrier liturgique: les variations climatiques d'un pays à l'autre font que les mêmes événements ne coïncident pas nécessairement quant aux dates. D’autre part, et dans le cas qui nous occupe ici, il y a peu de similitudes entre les agricultures espagnole et française. Certes, il y a des éléments communs. Ainsi, Santa Lucía, mengua la noche y crece el día équivaut grosso modo à A la saint Luce, le jour croît du saut d'une puce, et Lluvia suave quita vendaval correspond à Petite pluie abat grand vent. Mais avec Por San Blas/La cigüeña verás/Y si no la vieres/Año de nieves lie la disparition/ continuation du froid au retour éventuel des cigognes pour la Saint Blaise (3 février). Corrélation ignorée du français A la Saint-Blaise/L'hiver s'apaise/Mais s'il reprend/ Longtemps on s'en ressent. Que penser de En mayo, no te quites el sayo qui recommande de se méfier des premiers beaux jours, alors que le français prétend à l'inverse que En mai, fais ce qu'il te plaît! L'écart se creuse encore plus dans le domaine agricole, les cultures et l'élevage des deux pays respectifs présentant de grandes divergences. Là où le français affirme par exemple que À la Sainte-Catherine, tout prend 
racine (25 novembre), l'espagnol ne dit rien. Voici un cas où l'on peut cependant s'en tirer. Un des derniers dictons encore présents dans le parler quotidien est $\grave{A}$ la SaintRémi, cueille tes fruits ( $1^{\text {er }}$ octobre), distique a(5) a(3) recommandant donc la cueillette des fruits pour ce jour précis. L'espagnol voit le mois d'octobre en général comme plutôt le mois des semailles et surtout du retour du mauvais temps. À bien y regarder, il existe cependant des phrases sentencieuses relatives à la cueillette des fruits, à savoir: A últimos de noviembre, coge tu oliva siempre ('À la fin novembre, toujours ramasser les olives'); Agosto madura, y septiembre vendimia la uva ('Août mûrit, et septembre vendange'); Agosto tiene la culpa, septiembre tiene la pulpa ('Août a la coulpe, septembre a la pulpe’); Por San Francisco, todo fruto es exquisito' ('À la SaintFrancis, tout fruit est exquis'). Les deux premières ne conviennent guère: la première (distique a(8) a(8)) est relative au mois de novembre, et non au $1^{\text {er }}$ octobre, et concerne uniquement les olives. La seconde n'a trait qu'à la vendange, et parle de septembre. La troisième, également un distique $\mathrm{a}(8) \mathrm{a}(8)$, dit que les fruits en général sont à point en septembre, mais ne parle pas du premier octobre. La dernière enfin, un tercet Por San Francisco/Todo fruto/Es exquisito $=\mathrm{a}(5) \mathrm{b}(4) \mathrm{a}(5)$, mentionne explicitement les fruits, et la Saint-François a lieu le 4 octobre. Dans un contexte très général, c'est donc cette dernière qui conviendra le mieux, les autres pouvant par ailleurs convenir pour des correspondances plus spécifiques de date ou d'objet.

Je voudrais terminer par l'exemple d'un proverbe espagnol très célèbre, pour lequel il n'existe rien d'équivalent en français contemporain, ce qui va nous obliger à adopter des solutions quelque peu désespérées. Il s'agit de Más tiran dos tetas que cien carretas, littéralement 'Plus tirent deux mamelles que cent charrettes'. D'utilisation courante, la leçon ci-dessus est un tercet /Más tiran/dos tetas/ que cien carretas/, de structure $a(3) b(3) b(5)$, qui possède de très nombreuses variantes, dont certaines fort lestes, et qui est très anciennement attesté ${ }^{32}$. Ce proverbe exprime dans un style familier le pouvoir de domination que le désir sexuel permet à la femme d'exercer sur l'homme. Or, le français, malgré un grand nombre de phrases sentencieuses consacrées en bien ou en mal à la femme, ne possède rien qui ressemble à la phrase espagnole, même de loin. Il a pourtant existé en ancien français un Plus tire cus que corde $e^{33}$, proche de certaines variantes du proverbe espagnol ${ }^{34}$, mais hélas, disparu aujourd'hui: l'idée - et l'image - n'est donc pas étrangère au champ parémique français. D'autant moins que La Curne dans son Dictionnaire cite un Un cheveu de ce que l'on aime tire plus que quatre boufs, qui exprime une idée très proche. Pour nous tirer d'affaire, je rappellerai une remarque faite plus haut: les sujets parlants identifient souvent un proverbe non à son contenu, mais à sa structure rythmique. Si la structure est adéquate, la forme est interprétée comme un proverbe d'une autre région, ou une variante dialectale. L'idée ici sera donc de forger de toutes pièces une phrase parémique correspondant à l'idée de la phrase espagnole, en lui conférant une structure rythmique qui la fera interpréter comme un authentique proverbe. Pour traduire tetas, mot familier mais non nécessairement vulgaire, j'utiliserai tétons, que je ferai rimer avec charreton. L'idée est de reconstituer ainsi un tercet en respectant le maximum d'équivalences. La traduction de Más tiran par 'tirent plus', comme dans la citation de La Curne est faible: l'espagnol tirar signifie ici 'tirer de façon irrésistible, attirer fortement', ce pour quoi je suggérerai la traduction 'tirer plus fort'. Ce qui nous donne la phrase parémique Deux tétons tirent plus fort que cent charretons, générique et métaphorique, et pourvue de plus d'un schéma rythmique /Deux tétons/ 
tirent plus fort/que cent charretons/ = a(3) b(3) a(4) proche de l'original $\mathrm{a}(3) \mathrm{b}(3) \mathrm{b}(5)$. On peut bien entendu signaler le côté marginal de la traduction en l'assortissant d'un commentaire du style de Comme on dit chez moi. On sauve ainsi l'esprit du texte parémique original - exigence minimale de toute traduction - mais on satisfait également certaines exigences propres au domaine des formes sapientiales.

\section{NOTES}

1. Cf. infra.

2. Livre des proverbes français, Hachette, Paris, 1996.

3. Il me faut quatre pneus, les voitures ont quatre roues/Les voitures ont quatre roues, il me faut quatre pneus.

4. Outre comme on dit, les plus courants sont comme dit la sagesse populaire, comme dit l'autre, comme on dit chez moi, et On sait bien que;

5. Mais comme le disait le proverbe espagnol, il ne faut pas vendre la peau de l'ours avant de l'avoir tué...

6. À l'origine de cette notion, Berrendonner (1981).

7. J'utiliserai [adage] pour les phrases parémiques non métaphoriques à contenu moral ou juridique, [dicton] pour celles concernant le rapport avec la nature.

8. Une telle structure - combinaison d'heptasyllabes et de pentasyllabes - est très fréquente dans le domaine parémique, mais également dans le domaine poétique: ainsi le haïku et le tanka (japonais), mais aussi la seguidilla (espagnole). En français, le phénomène est moins net, peut-être à cause de l'extraordinaire succès de l'alexandrin.

9. Les combinaisons de ce type sont très communes, les plus courantes étant 7/5, 6/4, et 5/3.

10. Le basque semble avoir également suivi ce modèle: Ainhara batek ez du udaberria egiten, lit. 'hirondelle/une/ non/il a/printemps le/fait' (dialecte labourdin). Les variations selon les langues entre 'printemps' et 'été' ne viennent pas de l'observation de variations climatalogiques, mais d'une erreur de traduction. En latin, prima vere était la première partie du printemps, et veranum tempus la seconde partie, l'été étant désigné par aestivum tempus. D’où le doublet espagnol verano/estío.

11. Lit. 'Chien aboyeur, peu mordeur'.

12. C'est d'ailleurs le sens officiel que lui donne la Real Academia Española.

13. Désireux de ne pas entrer dans des détails de technique linguistique qui n’ont pas leur place ici, je me contenterai d'un survol rapide des différents modes de fonctionnement. Les faits sont plus compliqués, et on peut opérer des distinctions plus fines.

14. Lit. 'Il y a chat enfermé'.

15. Lit. 'Eau passée ne meut pas moulin'.

16. Lit. 'La thune, c'est la thune'.

17. Sur ce sujet, cf. Anscombre (2001, 2003, 2006 [à paraître]).

18. 'Le droit est foulé au pied quand domine la force'.

19. À savoir le Marqués de Santillana, Refranes que dicen las viejas tras el fuego, 1454.

20. Lit. 'Quand la rivière fait du bruit, c'est qu'elle charrie de l'eau'.

21. Il s'agit là d’un fait général. Ainsi, la très utilisée Quien fue a Sevilla, perdió su silla ('Qui va à la chasse perd sa place') cède de plus en plus de terrain devant la version au présent Quien va a Sevilla, pierde su silla.

22. Ainsi le peu courant Jamais bon cheval ne devint rosse, ou l'espagnol Quien tuvo, retuvo .

23. Lit. 'Entre du chou et du chou, de la laitue'.

24. Recensé comme proverbe turc par Montreynaud-Pierron-Suzzoni.

25. Lit. 'Mieux vaut un 'Prends' que deux 'Je te donnerai' .

26. Lit. 'Il ne faut pas vendre la peau de l'ours avant de l'avoir chassé', moins courant qu'en français.

27. Lit. 'Il ne faut pas manger le lapin avant de l'avoir chassé'.

28. Sur ces questions, cf. Anscombre (2000a et 2000b) pour un aperçu rapide et non technique.

29. La lettre indique le type de la rime, et le chiffre le nombre de syllabes, de "pieds».

30. Lit. «Même si la guenon s'habille de soie, guenon elle reste.»

31. Lit. 'Avec la beauté seule, on ne met pas la marmite'.

32. Dès le $\mathrm{XVI}^{\mathrm{e}}$ siècle.

33. Proverbes au vilain, manuscrit, fin $\mathrm{XIII}^{\mathrm{e}}$.

34. En particulier Más tira coño que soga et Más tiran nalgas en lecho que buyes en barbecho. 


\section{RÉFÉRENCES}

Anscombre, J.C. (1997): "Reflexiones críticas sobre la naturaleza y el funcionamiento de las paremias”, Paremia 6, pp. 43-54.

Anscombre, J.C. (1999): “Estructura(s) métrica(s) en los refranes", Paremia 8, pp. 25-36.

Anscombre, J.C. (2000a): «Parole proverbiale et structures métriques», Langages 139, pp. 6 26.

Anscombre, J.C. (2000b) : «Folklore, folklore. Est-ce que j'ai une gueule de folklore? », L'Observatoire de la Télévision 18, pp. 25-27.

Anscombre, J.C. (2001) : "Refranes, polilexicalidad y expresiones fijas", in Actas del IV Congreso Internacional de Lingüística Francesa, Santiago de Compostela (23-25 septembre 1999).

Anscombre, J.C. (2003): «Les proverbes sont-ils des expressions figées?», Cahiers de lexicologie 1 , pp. 159-173.

Anscombre, J.C. (2006): «Les proverbes: un figement du deuxième type?», Linx, Paris.

Aquiem, M. (1993): Dictionnaire de poétique, Guides de la langue française, coll. «Le livre de Poche», Paris.

Barella, A. y J.G. Campos (1993): Diccionario de refranes, Espasa Calpe, Madrid.

Berrendonner, A. (1981): La pragmatique, Ed. de Minuit, Paris.

Cerquiglini, J. et B. Cerquiglini (1976): «L'écriture proverbiale», Revue des sciences humaines 163, pp. 359-375.

Сомвet, L. (1996): «Los refranes: origen, función y futuro», Paremia 5, pp. 11-22.

Conde TARrio, G. (1997): Estudio comparativo de las paremias en francés, castellano y gallego, tesis doctoral, Universidad de Santiago de Compostela.

Delacourt, F. (1996): Proverbes, dictons, et citations, Ed. de Vecchi S.A., Paris.

Desruisseaux , P. (1997): Le petit proverbier, Ed. Pierre DesRuisseaux, Québec.

Djavadi, C. (1990): Rouge du soir. Dictionnaire des dictons météorologiques, Ed. Christian, Paris.

Dournon, J. (1986): Le dictionnaire des proverbes et dictons de France, Hachette, Paris.

Garcia Peinado, M.A. (1993): «Le fonds populaire chez La Fontaine: proverbes et locutions proverbiales", Paremia 2, pp. 195-198.

Gimeno, I. (1989): El llibre dels refranys catalans, Edicions Cap Roig, Barcelona.

Gonzalez, J.L. (1998): Dichos y proverbios populares, Edimat, Madrid.

Gross, G. (1996): Les expressions figées en français, coll. «L’essentiel», Ophrys, Paris.

Guiter, H. (1969): Proverbes et dictons catalans, R.Morel Ed., France.

JunCEDA, L. (1998): Diccionario de refranes, Espasa Calpe S.A., Madrid.

KLeiber, G. (1989): «Sur la définition du proverbe», coll. «Recherches Germaniques», n², pp. 232-252.

Le Roux De Lincy (1996/1842): Le livre des proverbes français, Hachette, Paris.

Lis, M. et M. BARbier (1980): Dictionnaire du gai parler, Mengès, Paris.

Maloux, M. (1995/1980): Dictionnaire des proverbes, sentences et maximes, Larousse, Paris.

Mendez Perez, A. (1996), "Mnemotecnia del refrán. La rima y las estructuras", Paremia 5, pp. 183-186.

MejRI, S. (1996): Le figement lexical, thèse d'État, Université de Tunis.

Milner, B. (1969): «De l'armature des locutions proverbiales. Essai de taxonomie sémantique», L'Homme, Juillet-Septembre, pp. 4-70.

Montreynaud, F., A. Pierron et F. Suzzoni (1980): Dictionnaire des proverbes et dictons, Les Usuels de Robert, Robert, Paris.

Morawski, J. (1925): Proverbes français antérieurs au XVe siècle, París, Champion.

Navar ro Dominguez, F. (1993): "Hacia una nueva caracterización del concepto de paremia en su empleo lingüístico-discursivo", Paremia 2, pp. 21-26.

Pares i Puntas, A. (1997): Diccionari de refranys Català-Castellà/Castellà-Català, El Cangur 240, Edicions 62, Barcelona.

Perez Martinez, H. (1997): Refrán viejo nunca miente, El Colegio de Michoacán, México. 
268 MetA, LIII, 2, 2008

Permjakov, G. L. (1984): "Structural Typology of Paremias", Kodikas/Code 7-3/4, pp. $263-$ 268.

Pierron, A. (1997): Dictionnaire des proverbes, Marabout, Alleur.

Quilis, A. (1994): Métrica española, Ariel, Barcelona.

Rey, A. et S. Chantreau (1997): Dictionnaire d'expressions et locutions, Le Robert, Paris.

Sevilla-Muñoz, J. (1988): Hacia una aproximación conceptual de las paremias francesas y españolas, Madrid, Editorial Complutense.

Sevilla-Muñoz, J. (2001): 1001 refranes españoles con su correspondencia en alemán, árabe, francés, inglés, italiano, polaco, provenzal y ruso, Ediciones Internacionales Universitarias, Madrid.

Simpson, J. (1982): The Concise Oxford Dictionary of Proverbs, Guild Publishing London, Londres.

TамвA, I., (2000): «Formules et dire proverbial», Langages 139, pp. 110-118. 